\title{
Andrea AUGENTI, Città e porti dall'Antichità al Medioevo
}

\section{Henri Bresc}

\section{CpenEdition \\ Journals}

Édition électronique

URL : https://journals.openedition.org/ccm/6758

DOI : $10.4000 / \mathrm{ccm} .6758$

ISSN : 2119-1026

\section{Éditeur}

Centre d'études supérieures de civilisation médiévale/Université de Poitiers

\section{Édition imprimée}

Date de publication : 1 janvier 2021

Pagination : 61-63

ISBN : 978-2-490783-08-3

ISSN : 0007-9731

\section{Référence électronique}

Henri Bresc, «Andrea Augenti, Città e porti dall'Antichità al Medioevo », Cahiers de civilisation médiévale [En ligne], 253 | 2021, mis en ligne le 01 mars 2021, consulté le 23 novembre 2022. URL : http:// journals.openedition.org/ccm/6758 ; DOl : https://doi.org/10.4000/ccm.6758

\section{(c) $)(9)$}

Creative Commons - Attribution - Pas d'Utilisation Commerciale - Pas de Modification 4.0 International - CC BY-NC-ND 4.0

https://creativecommons.org/licenses/by-nc-nd/4.0/ 


\section{COMPTES RENDUS}

Andrea Augentr, Città e porti dall'Antichità al Medioevo, Rome, Carocci editore (Aulamagna, 49), 2018.

Pour une collection de manuels destinés à l'enseignement supérieur, Andrea Augenti, archéologue du port romain de Classe et enseignant à l'université de Bologne à Ravenne, a préparé un ouvrage d'initiation et de réflexion sur l'archéologie des ports méditerranéens et des emporia de la Manche, de la mer du Nord et de la Baltique entre le $\mathrm{vI}^{\mathrm{e}}$ et le $\mathrm{IX}^{\mathrm{e}} \mathrm{s}$. Sous ce titre ambitieux, son dessein était d'ouvrir à ses collègues et aux étudiants un espace lointain, des publications de fouilles principalement en anglais ou dans les langues scandinaves et de répondre aux questions que pose le livre fondateur d'Henri Pirenne, Mahomet et Charlemagne. C'est un petit livre de 179 p., 160 de texte (dont 65 d'illustrations), une bibliographie en effet précieuse, et des index. Le style en est familier et agréable, il fait voyager son lecteur de port en port, avec une prédilection pour les vues reconstituées de villes et de maisons à la mode des bandes dessinées. Le livre sera utile, avec quelques réserves sur ses raccourcis : p. 144, il attribue des «origines flamandes» à Henri Pirenne. On sait que, né à Verviers, le grand historien se voulait avant tout belge et il avait dû quitter l'université de Gand en 1930 lors de sa «flamandisation» faute de parler le néerlandais. Autre petite réserve : sur les illustrations, également précieuses, tirées des publications de fouilles, les indications sont portées en corps minuscule et peu lisibles.

Le point de départ et la conclusion portent sur la validité, aujourd'hui, des thèses d'H. Pirenne. L'a. rappelle que débat a été vif et qu'un consensus s'est établi sur le déclin lent de l'activité commerciale de la Méditerranée romaine au $\mathrm{VI}^{\mathrm{e}} \mathrm{s}$., sur une inflexion brutale au VII ${ }^{\mathrm{e}}$, un nadir au $\mathrm{VIII}^{\mathrm{e}}$, tandis que le désaccord subsiste sur une deuxième question, le moment d'un autre point d'inflexion, celui de la réanimation, entre $\mathrm{VIII}^{\mathrm{e}}$ et IX $\mathrm{I}^{\mathrm{e}} \mathrm{s}$. Cette problématique raccorde l'ouvrage à un grand débat, souvent truqué par l'obstination des historiens à imposer le mythe d'un «printemps» productif et commercial de l'Islam, et A. Augenti ne répond pas, en partie faute de sources archéologiques, à cette deuxième interrogation, qui excède sans doute les limites temporelles fixées à l'ouvrage.

La référence à ce débat permet d'introduire une première synthèse sur la mondialisation romaine, entre $\mathrm{II}^{\mathrm{e}}$ et $\mathrm{VII}^{\mathrm{e}} \mathrm{S}$., fondée en particulier sur la production agricole et l'industrie céramique de l'Afrique romaine, et sur le déclin des ports de Méditerranée : Carthage, Césarée de Palestine; les ports de Rome, Ostie et Porto, Classe, et ceux de de Ravenne et Marseille. Tous connaissent un déclin brutal, l'envasement et l'abandon au VII' s., sauf Porto dont l'activité, le ravitaillement de Rome, se maintient quelque temps, jusqu'à l'occupation de la Sicile par les Aghlabides et encore au-delà, et Marseille, qui conserve sous les Mérovingiens son rôle d'escale des produits du Levant. Un problème se pose cependant au médiéviste à la vision de leurs plans : ces ports sont petits, minuscules même Carthage, qui ne pourrait assurer qu'une part mineure de l'énorme exportation d'huile, de vin, de blé, de céramique de l'Afrique romaine. Le système portuaire y a nécessairement compris un grand nombre d'embarcadères, scale, caricatori, calanques ou plages, à proximité des latifundia, où le chargement, nécessairement long, n'implique pas un déplacement à longue distance. Dans les avant-ports des capitales, Rome et Ravenne, au contraire, le déchargement et le stockage sont rapides. Un chantier de recherches sur les embarcadères s'offre donc à l'archéologue.

Une deuxième synthèse envisage l'autre volet de l'enquête archéologique, celui des emporia, qui portent le nom de wic, dont les résultats des fouilles sont rapidement condensés. Ils sont dix, dont les dates extrêmes de l'activité sont données par le matériel : Dorestad en Frise (635-860), Quentovic à l'embouchure de la Canche (VII' s.-842), Hamwic (près de Southampton) qui a connu son apogée v. 850, Gipeswic (Ipswich) dont la vie s'est maintenue au $\mathrm{IX}^{\mathrm{e}} \mathrm{s}$., Lundenwic (à un $\mathrm{km}$ à l'ouest de la cité romaine 
de Londres, 680-866), Eoforwic (York, au S.-E. de la cité romaine), Ribe (VIII ${ }^{\mathrm{e}}$ s. -900$)$ et Hedeby $\left(750-\mathrm{x}^{\mathrm{e}} \mathrm{s}\right.$.) dans le Jutland, Birka (750-fin du $\mathrm{x}^{\mathrm{e}} \mathrm{s}$.) en Suède, et Kaupang (800-930/950) dans le fjord d'Oslo. L'enquête a commencé tôt, autour de 1850 dans les pays scandinaves, dont les premières villes sont un élément fondateur de l'histoire nationale, et les emporia ont été des incubatrices, des chantiers d'expérimentation de techniques archéologiques nouvelles : fouille extensive partout, récolte et exploitation des tissus, des restes osseux, des grains, des charbons, prospection géophysique et sondage non destructif à Quentovic.

Les conclusions sont rassemblées p. 92-105: les emporia connaissent une vie brève, un à deux siècles, des dimensions et une population variables, toujours modestes, par rapport aux ports du monde méditerranéen à leur apogée, une planification par lots rectangulaires qui accueille un développement urbanistique original, maisons et infrastructures de bois (cuvelage des puits, pavement des rues, quais et appontements), sans murs de défense ni monuments, des cimetières très diversifiés, de la crémation à l'ensevelissement, aux barques funéraires. L'a. en déduit des caractères généraux : fondation autoritaire, probablement par les jeunes monarchies germaniques, population socialement et «ethniquement» (mais le mot est ambigu : race, peuple, culture ?) très variée, vocation fonctionnelle, commerce et artisanat, qui débouche sur des «non-lieux», sans monuments ni mémoire. Les matériels analysés confirment d'abord le dessein supposé des rois : assurer un marché d'importation de produits lointains, établir un centre douanier et un hôtel des monnaies. On frappe en effet monnaie à Dorestad depuis 635-640, à Ribe depuis 720 et à Hedeby. Il ne s'agit donc pas de marchés connectifs, mais de commerce à longue distance.

Des nuances sont cependant sensibles : deux des sites fouillés manifestent une première phase sans édifices construits dans les parcelles rectangulaires établies et bornées perpendiculaires à la plage, Ribe et Kaupang. Il s'agit donc de marchés non permanents, saisonniers et explosifs, comme on en observait encore récemment dans l'Atlas marocain où ils ont été étudiés par Francisco Benet. Il faut également corriger l'idée d'absence générale de monuments et de «non-lieu» universels : deux des dix emporia sont des faubourgs à faible distance de villes romaines et on a construit une église à Hamwic, en bois sans doute, qui apparaît sur la reconstitution du paysage urbain, dessinée à partir du plan de fouille p. 67 . Les sites étudiés connaissent donc un cycle de transformations; ils ont pu être assassinés, selon la formule de Piganiol, en pleine évolution ou achever la mission qui leur avait été confiée et mourir de leur belle mort. Leur état, au moment où l'abandon les fige, relève d'une histoire événementielle, comme dans toutes les fouilles. La date des abandons ou des replis sur l'habitat fortifié (Londres, York) restaure la validité de l'hypothèse d'un rôle décisif des raids vikings : un premier temps de pillage et de destruction, avant celui de l'exploitation méthodique et des commerces, comme dans l'histoire parallèle des conquêtes musulmanes. Les emporia scandinaves ont évidemment une durée de vie et d'activité beaucoup plus longue. Leur rôle éventuel dans la formation des flottes vikings et dans la redistribution des butins n'est cependant pas évoqué. Dans une économie où le vol et le don, Gift and Thieft, sont un volet des commerces, c'est un point à approfondir. On en a les prémices dans la présence précoce en Scandinavie des dirhams d'argent de l'Orient islamique, où un peu de commerce (ambre, peausseries) se combine avec le mercenariat et les pillages avant la fondation de l'État.

Les fossiles directeurs (céramiques rhénanes du type Badorf et du type Tating, céramique neustrienne de La Londe, verre rhénan et meules de basalte de Niedermendig) et l'abondance de l'argent monnayé (protopennies ou sceattas) illustrent le grand commerce maritime à longue distance, que confirme la situation des emporia sur les plages, au fond des embouchures ou sur les lacs qui communiquent avec la Baltique. Une autre céramique, du type Ipswich, diffusée dans un rayon de $180 \mathrm{~km}$ autour de l'emporium commercial et artisanal (carte p. 118), permet l'hypothèse d'un réseau de foires, de sites miniers et de productions artisanales dispersées dans le territoire, que de premières fouilles et l'usage - légalisé en Grande-Bretagne - de détecteurs de métaux ont permis d'esquisser la carte. On se méfiera de cet enthousiasme pour ce dernier outil qu'on sait dévastateur en de mauvaises mains, comme de la passion pour le graphe qui crée l'illusion : un croquis est particulièrement trompeur, p. 108, emprunté à Michael McCormick, sur le commerce des esclaves. Un petit nombre de flèches portées sur une carte à grande échelle donne l'impression de flux constants et massifs d'Angles, puis de Slaves vers le monde méditerranéen et une comparaison implicite avec le rôle prétendu de la traite océanique dans l'accumulation du capital au XVIII' $\mathrm{s}$. permet d'accepter l'affirmation, p. 108, que c'est le commerce invisible des esclaves - sans traces archéologiques - qui a fait décoller le protocapitalisme. Un peu de prudence s'impose. La revanche inattendue de la connectivité, terrestre pour l'essentiel (les sites de plage montrent qu'on y 
venait aussi par barque), suppose une économie plus organisée, hiérarchisée, de vraies régions construites autour des emporia et qui survivent aux guerres et aux invasions : A. Augenti met en rapport des sites miniers et artisanaux anglo-saxons avec des abbayes qui les ont protégés par-delà la destruction du centre marchand.

On débouche sur deux modèles : Richard Hodges proposait trois types de sites (A pour de simples plages, B pour un marché nodal avec habitat structuré, $\mathrm{C}$ pour un centre administratif et résidentiel) et Dagfinn Skre nuance le tableau en ajoutant une résidence aristocratique flanquée d'un marché et un marché local qui cristallise l'artisanat. Frans Theuws, partant du concept de «non-lieu», introduit une nécessaire dimension religieuse : la présence du monument, d'un «sacré» indéfini, d'un monastère en particulier, contribue à protéger et perpétuer le site. Laissons ce concept de sacré, flou et trompeur, et qui sousentend des continuités entre paganisme et chrétienté. La christianisation est au contraire décisive : reliques et pèlerinages définissent un pôle de la grâce, besoin nouveau, avec des accès permanents et saisonniers, occasions de foires (Lendit, Maastricht, Saint-Bavon de Gand), tandis que des églises de bois, puis de pierre, des tombes familiales regroupées autour d'elles, ont partout coagulé l'habitat autour de cette double mémoire. La survie des emporia dépend donc de la présence du roi et/ou de la protection qu'offre le saint.

Un chapitre original pose des questions nouvelles : l'espace adriatique oppose le déclin et l'abandon, au $\mathrm{VII}^{\mathrm{e}} \mathrm{S}$. de la cité de Buthrinte, sur la côte épirote de l'Albanie moderne, après une remarquable prospérité au $\mathrm{VI}^{\mathrm{e}} \mathrm{S}$., marquée par la construction de monuments, et l'essor simultané de Comacchio, sur la lagune vénitienne. La première est victime de la rétraction générale de l'Empire, avant et pendant les invasions arabes et les guerres longobardes. Comacchio, en revanche, ravitaille le royaume de Liutprand en sel et en produits d'importation de l'Orient byzantin, avant d'être détruite par les Vénitiens, ses concurrents. A. Augenti voit dans cet emporium méditerranéen le point de départ d'une histoire qui contrebalance l'essor du monde de la mer du Nord et de la Baltique. On peut aussi l'analyser comme un autre portail d'un monde barbare unique dont l'essor économique est soutenu par l'initiative de monarchies fascinées par les modèles impériaux, fragiles et qui craquent au $\mathrm{X}^{\mathrm{e}} \mathrm{s}$., et par des facteurs plus durables, le dynamisme agricole, la demande de l'aristocratie et de l'Église. L'a. pose une question finale, encore sans réponse : pourquoi les sites méditerranéens sont-ils si pauvres de monnaies?

Une analyse complète de l'apport d'H. Pirenne, au-delà de son rôle de moteur dans l'analyse des emporia, aurait dû tenir compte de la coupure effective de la Méditerranée et de l'abandon des routes maritimes : la fouille de Santa Maria del Mare (Squillace), en Calabre, a montré l'absence de céramique provenant de la Sicile musulmane au $\mathrm{IX}^{\mathrm{e}} \mathrm{S}$. et la rupture d'une connectivité encore vive, tandis que Malte ravagée et dépeuplée, reste vide pendant plusieurs siècles, de 870 à 1048-1049. La flotte musulmane existe bien, capable d'expéditions à long rayon, la mer n'est pas morte, mais ce sont des fonctions guerrières qui sont assumées, jusqu'au front de carrière géré en particulier par l'émirat de Bari et qui assure l'extraction violente d'esclaves en Italie méridionale.

Le petit manuel d'A Augenti peut servir de guide aux historiens français; écrit dans un italien simple, il se lit facilement et c'est une approche imparfaite sans doute, un survol et des balises, mais la dimension de sa synthèse interdisait une profondeur anthropologique. Il cite ainsi Stéphane Lebecq : le marchand naît au VIII ${ }^{\mathrm{e}}$ s., rassemblé en une confrérie protectrice. Auparavant et bien après aussi, une autre figure parcourt ce haut Moyen Âge, le marchand ambassadeur, porteur de cadeaux, espion, otage, membre de colonies étrangères au rôle ambigu, celui des «ports de commerce» de Karl Polanyi et de ses élèves. L'échange marchand n'est qu'une part du mouvement des marchandises, tandis que la neutralité politique s'impose en marge de l'État en formation. Autant de pistes déjà balisées et qui offriront du grain à moudre à l'interprétation des fouilles de ports.

Henri BRESC Université de Paris X-Nanterre 